\title{
Acknowledgement of manuscript reviewers 2018
}

\author{
James Elliott Scott ${ }^{1,2}$, Israel Agaku
}

\section{CONTRIBUTING REVIEWERS}

The editors of Tobacco Induced Diseases would like to thank all our reviewers who have contributed to the journal in Volume 16 (2018).

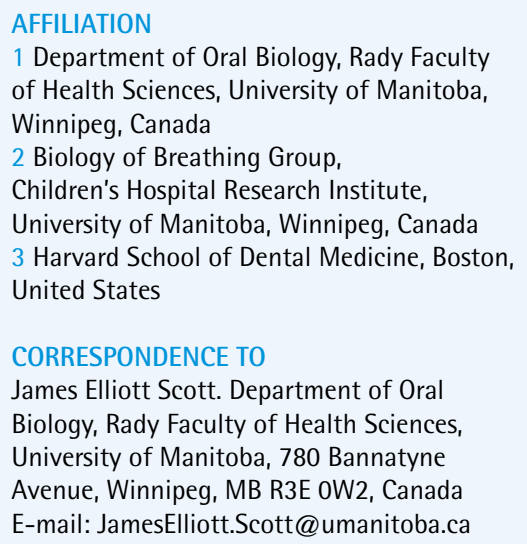

\section{Montse Ballbè}

Spain

Sri Lanka

Tatiana Andreeva

Ukraine

Davut Baltaci

Turkey

\section{Amitav Banerjee}

India

$\begin{array}{ll}\text { José Antunes } & \text { India } \\ \text { Brazil } & \end{array}$

Yael Bar-Zeev

Australia

\section{Arzu Beklen \\ Turkey}

$\begin{array}{ll}\text { Francisco Ayesta } & \text { Helen Binns } \\ \text { Spain } & \text { United States }\end{array}$

$\begin{array}{ll}\text { Francisco Ayesta } & \text { Helen Binns } \\ \text { Spain } & \text { United States }\end{array}$
Fatmah R. Ali

United States

Qasem Alomari

Kuwait
Raed Bahelah

Yemen

Sabeeh A. Baig

United States

\section{Linda Bledsoe}

United States

Pinar Bostan

Turkey 
Marc T. Braverman

United States

Leslie Ann Brick

United States

Betsy Brock

United States

Jennifer Brown

United States

Nurcan Buduneli

Turkey

Karen Calabro

United States

Priscilla Callahan-Lyon

United States

Natalie Capps

United States

Ralph Caraballo

United States

Victor Cardenas

United States

Timothy Chambers

New Zealand

Po-Yin Chang

United States

Omar Chehab

Lebanon

Julia Chen

United States

Onyema Chido-Amajuoyi

United States

Parimal Chowdhury

United States
Fatma Cihan

Turkey

Nursan Cinar

Turkey

Bradley Collins

United States

Mark Conner

United Kingdom

Nancy Tess Boley Cruz

United States

Dardo Curti

Uruguay

Nazir A. Dar

India

Mike Daube

Australia

Bertrand Dautzenberg

France

Richard Daynard

United States

Silvio De Flora

Italy

Dennis de Ruijter

Netherlands

Olufemi Olumuyiwa Desalu

Nigeria

Ligia Devóglio

Brazil

Yuan Di

Australia

Amanda Dickinson

United States
Jeremy E. Drehmer

United States

Michaela Dušková

Czech Republic

Sarah Edwards

Canada

Marwan E. El-Sabban

Lebanon

Ubiracé Fernando Elihimas Jr

Brazil

Edward Ellerbeck

United States

Lucinda England

United States

Mukremin Er

Turkey

Ebru Erdemir

Turkey

Nazmiye Erdogan

Turkey

Daniel Erku

Ethiopia

Patricia Escobedo

United States

William Evans

United States

Changyong Feng

United States

Filippos Filippidis

United Kingdom

Elizabeth Flott

United States 
Omid Fotuhi

Canada

Diane Francis

United States

Kate Frazer

Ireland

Deborah Fritz

United States

Erika Froelicher

United States

Silvia Fustinoni

Italy

Silvano Gallus

Italy

Esther Garcia-Esquinas

Spain

Gladwell Gathecha

Kenya

Patrick Geraghty

United States

Wan Ghani

Malaysia

Laura Gibson

United States

Alexander Gilkes

United Kingdom

Gary A. Giovino

United States

Charis Girvalaki

Greece

Rebecca Glover-Kudon

United States
Tatiana Goerig

Germany

Giuseppe Gorini

Italy

Trisha Greenhalgh

United Kingdom

Osman Günay

Turkey

Hakan Gunen

Turkey

Prakash Gupta

India

Joseph Guydish

United States

Takashi Hanioka

Japan

Wojciech Hanke

Poland

Alyssa Harlow

United States

Arusyak Harutyunyan

Armenia

Linnea Hedman

Sweden

Lisa Henriksen

United States

Gholamreza Heydari

Iran

Heikki Hiilamo

Finland

Rosemary Hiscock

United Kingdom
Sai Yin Ho

Hong Kong

David Homa

United States

Melbourne Hovell

Israel

Pei-Ting Hsu

Taiwan

Hsien-Liang Huang

Taiwan

Ruijie Huang

China

Karin Hummel

Netherlands

Muhammad Jami Husain

United States

Azmina Hussain

Pakistan

Sinem Iliaz

Turkey

Farhad Islami

United States

Ali Jawaid

Pakistan

Sun Jee

Republic of Korea

Nan Jiang

United States

Kevin John

United States

Nitin Joseph

India 
Ibrahim Ali Kabbash

Egypt

Steven Ndugwa Kabwama

Uganda

Sara Kalkhoran

United States

Gordana Kamceva

FYROM

Dilek Karadoğan

Turkey

Kamaleddin Karimyan

Iran

Kota Katanoda

Japan

Erinne Kennedy

United States

Georges Khalil

United States

Tamkeen Khan

United States

Joshua Kibet

Kenya

Daehyun Kim

Republic of Korea

Leon Kosmider

United States

Susi Kristina

Indonesia

\section{Allison Kurti}

United States

Sang Haak Lee

Republic of Korea
Sungkyu Lee

Republic of Korea

Yo Lee

Republic of Korea

Joshua B. Lewis

United States

Alex Liber

United States

Adam Lippert

United States

Feng Liu

China

Xiaoqiu Liu

Italy

Huai Loh

Malaysia

Tran Long

Australia

Brett Loomis

United States

Ángel López-Nicolás

Spain

Lucia Maria Lotrean

Romania

Liya Lu

United Kingdom

Mark Lucherini

United Kingdom

Ingeborg Lund

Norway

Dan Luo

China
James Macinko

United States

Renee Magnan

United States

Jeanne Mahoney

United States

Hadii Mamudu

United States

Michelle Manderski

United States

Lamberto Manzoli

Italy

Cristina Martínez

Spain

Kristy Marynak

United States

Mohammad Masjedi

Iran

Charu Mathur

United States

Yuuki Matsumoto

Japan

Debra McCallum

United States

Sam McCrabb

Australia

Anne Melzer

United States

Gülengül Mermer

Turkey

Alipasha Meysamie

Iran 
Stephen Miller

United States

Yoshiaki Minakata

Japan

Francis Mitrou

Australia

Laurenţiu Mogoantă

Romania

Jawad Mohammed

United Kingdom

Kahee Agid Mohammed

United States

Surapaneni Mohan

India

Ute Mons

Germany

Annie Montreuil

Canada

Meghan Moran

United States

Narine Movsisyan

Czech Republic

Donna Anne Murnaghan

Canada

Joshua Muscat

United States

Mark G. Myers

United States

Rima T. Nakkash

Lebanon

Jevae Nelson

United States
Kenneth Bruce Newbold

Canada

Nhung Nguyen

Vietnam

Fernando Nogueira

Brazil

Margaret Nolan

United States

Fariz Nurwidya

Japan

Satomi Odani

United States

Masaki Ohsawa

Japan

M.A.A. Oliveira Serra

Brazil

Sophie Orton

United Kingdom

Ellis Owusu-Dabo

Ghana

Pannee Pantaewan

Thailand

R. Steven Pappas

United States

Linda Pederson

United States

Nasheeta Peer

South Africa

Melinda Pénzes

Hungary

Michele Pergadia

United States
Irene Pericot-Valverde

United States

Armando Peruga

Chile

Andrew Pipe

Canada

Barbara Pizacani

United States

Kinga Polańska

Poland

Yayi Prabandari

Indonesia

Elena Raffetti

Italy

Deepa Raghavan

United States

Angela Ratsch

Australia

Sofia Ravara

Portugal

Martin Raw

United States

Mirjam Reutter

Germany

Luz Reynales Shigematsu

Mexico

Polosa Ricardo

Italy

Megan E. Roberts

United States

Paul Roman

United States 
Daniel Romer

United States

Craig Ross

United States

Hana Ross

South Africa

Renata Rossi e Silva

Brazil

Kathleen Ruff

Canada

Tobias Rüther

Germany

Maguy Saffouh EI Hajj

Qatar

\section{Banu Salepci}

Turkey

Jonathan Samet

United States

Rassamee Sangthong

Thailand

Kazunari Satomura

Japan

Benjamin Schüz

Germany

David Scott

United States

Arielle Selya

United States

Saul Shiffman

United States

Carlos Sillero

United Kingdom
Akansha Singh

India

Pranil Man Singh Pradhan

India

Nithat Sirichotiratana

Thailand

Nina Skavlan Godtfredsen

Denmark

Danielle Smith

United States

Matiwos Soboka

Ethiopia

Chandrashekhar Sreeramareddy

Malaysia

Gerry Stimson

United Kingdom

Elizabeth Stuyt

United States

Hua Su

United States

Walton Sumner

United States

Steve Sussman

United States

Girija Syamlal

United States

Chie Taniguchi

Japan

Mehmet Tecellioglu

Turkey

Mary Thompson

Canada
Steven Thomsen

United States

Philip Tonnesen

Denmark

Serena Tonstad

Norway

Antigona C. Trofor

Romania

Panagiotis N. Tsikouras

Greece

Anna Tzortzi

Greece

Bukola Usidame

United States

Juliana Uzeloto

Brazil

Eva Anne Marije van Eerd

Netherlands

Paul Vanderkam

France

Tord Vedøy

Norway

Michelangelo Vestita

Italy

Matteo Vitali

Italy

Paulo Vitória

Portugal

Nicholas J. Wagner

United States

Kelvin Man Ping Wang

Hong Kong 
Pingman Wang

Hong Kong

Yingning Wang

United States

Kenneth D. Ward

United States

Kenneth Warner

United States

Lynn Weber

Canada

David A. Welsh

United States
Jean Wong

Canada

Biao Xu

China

Gonghuan Yang

China

Ying Yang

China

Xiao-Hua Ye

China

Selen Yegenoglu

Turkey
Shaoman Yin

United States

Emilia Zainal Abidin

Malaysia

Fengyu Zhang

United States

Li Zhou

China

Wioleta Zielińska-Danch

Poland 